\title{
Performance Analysis of Slotted ALOHA Systems with Energy Harvesting Nodes and Retry Limit Using DTMC Model
}

\author{
Katsumi Sakakibara Yoji Nakata Kento Takabayashi \\ Department of Information and Communication Engineering \\ Okayama Prefectural University \\ 111, Kuboki, Soja, Japan \\ Email: \{sakaki, cd29029y, kent.hf\}@c.oka-pu.ac.jp
}

\begin{abstract}
We analyze performance of slotted ALOHA systems with energy harvesting nodes and retry limit. We assume that the capacities of data and energy buffer at a node are one packet and $E$ packets, respectively, and that one data packet transmission consumes one energy packet. The data and the energy packet arrival processes are modeled by independent Bernoulli processes. Under these assumptions, we develop a node-centric two-dimensional discrete time Markov chain model. Based on the equilibrium point analysis, we derive the fixed point equation with respect to the ratio of nodes transmitting a data packet. The accuracy of numerical results derived from the fixed point equation is verified by computer simulation. The numerical results indicate that throughput, the offered traffic and the discard probability roughly depend on the minimum of the data packet generation probability and the energy packet generation probability.
\end{abstract}

\section{INTRODUCTION}

$\mathbf{E}$ NERGY harvesting techniques have been attracting researchers' interest in minimization of nodes by removing batteries. For example, in Wireless Sensor Networks (WSNs), a huge number of such battery-less tiny nodes may be dispersed in a wide area. Each node may harvest their energy from environment. When a huge number of nodes with bursty traffic contend with one another for a common communication channel such as WSNs, a Medium Access Control (MAC) protocol plays an important role which can greatly influence performance of networks with or without the use of energy harvesting techniques [1].

Performance of MAC protocols with energy harvesting nodes has been extensively investigated in the literature. Moradian and Ashtiani [2] analyzed the maximum stable throughput of slotted ALOHA systems consisting of finite number of energy harvesting nodes. They constructed a node-centric twodimensional Discrete-Time Markov Chain (DTMC) model with $(j, x)$, where $j$ is the number of energy packets in the energy buffer and $x$ is the elapsed time for the next retransmission. Foss et al. [3] discussed stability conditions of slotted ALOHA systems with infinite population of energy

This work was partly supported by Japan Society for the Promotion of Science under Grant-in-Aid for Scientific Research (C) (KAKENHI No. 25420379) harvesting nodes from the system-centric viewpoint of a queueing network. The system is described by a two-tuple $(q, v)$, where $q$ and $v$ are the total number of data and energy packets in the system, respectively. Bae [4] analyzed the delivery ratio of slotted ALOHA systems with energy harvesting nodes under delay constraints. A node-centric twodimensional DTMC model $(W, E)$ was constructed, where $W$ is the elapsed sojourn time of the leading data packet in the data buffer and $E$ is the number of energy packets in the energy buffer. Notice here that no retry limit of unsuccessful data packet is considered in the above literature [2], [3].

In this paper, we analyze performance of slotted ALOHA systems consisting of energy harvesting nodes with retry limit [5] using a node-centric two-dimensional DTMC model. Then, the performance is analyzed in terms of throughput, average transmission delay and discard probability of data packet due to excessive retransmission trials.

\section{System Model}

Consider a slotted ALOHA system consisting of $N$ energy harvesting nodes contending for a common channel. Each node is equipped with not only a single data packet buffer but also an energy packet buffer of $E$-packet capacity. From the single-buffered assumption each node can store only one data packet. The length of data packet to be transmitted is assumed to be fixed to the unit length. The time axis is divided into slots which suffices for a single data packet transmission. The propagation delay between nodes and the common receiver is negligible. A data packet arrives independently at each node with probability $\lambda$ in a slot. A node harvests an energy packet with probability $\varepsilon$ in a slot. A node can transmit a data packet with probability $p$, if its energy buffer is not empty. One energy packet is consumed when a node transmits a data packet. We consider neither capture effects nor channel noise, so that a data packet transmission succeeds, only if no other data packets are transmitted simultaneously. All data packets involved in collision are to be retransmitted, until the number of retransmission trials including the first transmission reaches to the retry limit $L$. As an example, a system model with two energy harvesting nodes is depicted in Fig. 1. 


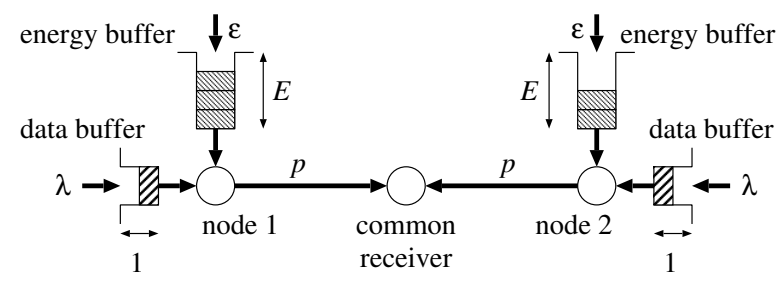

Fig. 1. System model of two energy harvesting nodes with single data buffer and with energy buffer of $E$-packet capacity.

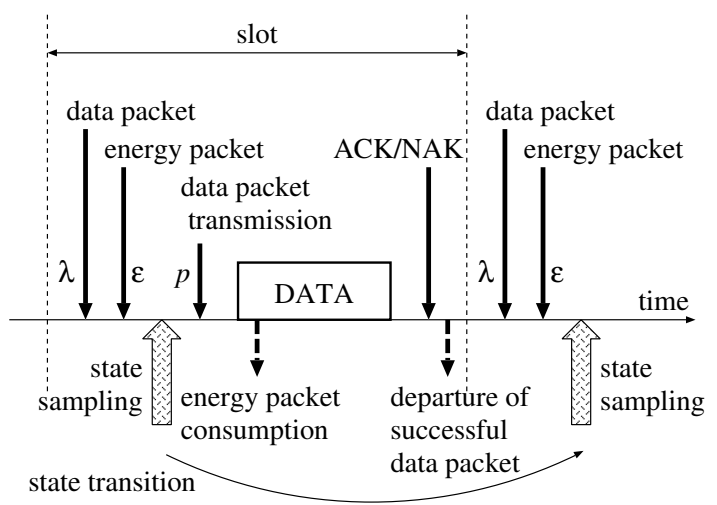

Fig. 2. Timing chart for state sampling.

\section{Discrete Time Markov Chain Model}

In order to accurately analyze the steady-state performance of the system, it is required to construct and solve a systemcentric DTMC model. However, it demands a considerably high dimensional DTMC model, which is complex to solve. Here, we take advantage of an equilibrium point analysis (EPA) [6], [7], which approximately evaluate the steady-state performance by using a node-centric DTMC model with an assumption that each node operates in an independent manner.

\section{A. State Sampling}

Consider a certain node. Let $i$ be the next number of transmission trials for a data packet in the node; $i=0,1,2, \ldots, L$. Note that a node with $i=0$ implies that it has no data packet. Let $j$ denote the number of energy packets in the node; $j=0,1,2 \ldots, E$. Then, from the single-capacity assumption for data packets, the state of each node in a slot can be described by a two-tuple $(i, j)$.

In order to facilitate construction of a DTMC model with respect to the state of a node, we first define the order of the stochastic events in a time-slot. As addressed in the previous section, there may be four stochastic events in a slot; data packet generation with probability $\lambda$, harvest of an energy packet with probability $\varepsilon$, data packet transmission with probability $p$ and outcome of data packet transmission; success or failure. At the end of a slot, a node which just transmitted a data packet receives a positive acknowledgment (ACK) or a negative acknowledgment (NAK).

\section{B. Two-Dimensional DTMC Model}

According to the sampling timing of the state, we can construct a node-centric two-dimensional DTMC model with respect to $(i, j)$ for $i=0,1, \ldots, L$ and $j=0,1, \ldots, E$. States $(i, j)$ for $i>0$ imply that a node is backlogged; that is, a data buffer at a node is occupied. States $(i, j)$ for $i>0$ and $j>0$ are those in which a node can transmit a data packet with probability $p$, since it has sufficient energy packets for data packet transmission.

\section{State Transition Probabilities}

State transition probability $p_{(i, j),(k, \ell)}$ from State $(i, j)$ to State $(k, \ell)$ can be obtained by taking into consideration the four stochastic events between two consecutive sampling points in Fig. 2;

1) data packet transmission at a backlogged node with nonempty energy buffer,

2) outcome of data packet transmission; success or failure,

3) data packet generation at a node with empty data buffer,

4) arrival of an energy packet.

First, suppose that a node has no data packet; that is, a node is in State $(0, j)$ for $j=0,1, \ldots, E$. If no data packet arrives and if an energy packet arrives, then a node moves to State $(0, j+1)$. We have state transition probabilities for thsese events;

$$
p_{(0, j),(0, j+1)}=(1-\lambda) \varepsilon
$$

for $j=0,1, \ldots, E-1$. If a data packet arrives without an energy packet, then state transition probabilities are

$$
p_{(0, j),(1, j)}= \begin{cases}\lambda(1-\varepsilon) & \text { for } j=0,1, \ldots, E-1 \\ \lambda & \text { for } j=E\end{cases}
$$

If both data and energy packets arrive, then we have

$$
p_{(0, j),(1, j+1)}=\lambda \varepsilon
$$

for $j=0,1, \ldots, E-1$.

Next, suppose that a node is backlogged. A state transition from $(i, j)$ to $(i, j+1)$ occurs, if a data packet is not transmitted and if an energy packet arrives. Then, we have

$$
p_{(i, j),(i, j+1)}= \begin{cases}\varepsilon & \text { for } j=0 \\ (1-p) \varepsilon & \text { for } j=1,2, \ldots, E-1\end{cases}
$$

for $i=1,2, \ldots, L$. Note that no data packet can be transmitted if a node is in State $(i, 0)$, since it has no energy. A node moves from State $(i, j)$ to State $(i+1, j-1)$, if a data packet transmission results in failure and if no energy packet arrives:

$$
p_{(i, j),(i+1, j-1)}=p P_{\text {fail }}(1-\varepsilon)
$$

for $i=1,2, \ldots, L-1$ and $j=1,2, \ldots, E$, where $P_{\text {fail }}$ is the probability of transmission failure of a data packet, which is formulated later. If a node fails in data packet transmission and if an energy packet arrives, then state transition probabilities are

$$
p_{(i, j),(i+1, j)}=p P_{\text {fail }} \varepsilon
$$


for $i=1,2, \ldots, L-1$ and $j=1,2, \ldots, E$. If a data packet transmission succeeds and if no data packet and no energy packet arrive, then a node transits from State $(i, j)$ to State $(0, j-1)$ :

$$
p_{(i, j),(0, j-1)}=\left\{\begin{array}{c}
p\left(1-P_{\text {fail }}\right)(1-\lambda)(1-\varepsilon) \\
\quad \text { for } i=1,2, \ldots, L-1 \\
p(1-\lambda)(1-\varepsilon) \text { for } i=L
\end{array}\right.
$$

for $j=1,2, \ldots, E$. Note that for $i=L$, a node moves from State $(L, j)$ to State $(0, j-1)$ when it transmits a data packet irrespective of success or failure. If only an energy packet arrives without a new data packet after successful data packet transmission, then we have

$p_{(i, j),(0, j)}= \begin{cases}p\left(1-P_{\text {fail }}\right)(1-\lambda) \varepsilon & \text { for } i=1,2, \ldots, L-1 \\ p(1-\lambda) \varepsilon & \text { for } i=L\end{cases}$

for $j=1,2, \ldots, E$. If a new data packet is generated without an energy packet generation after successful data packet transmission or after data packet discard, state transition probabilities are

$$
p_{(i, j),(1, j-1)}=\left\{\begin{array}{r}
p\left(1-P_{\text {fail }}\right) \lambda(1-\varepsilon) \\
\quad \text { for } i=1,2, \ldots, L-1 \\
p \lambda(1-\varepsilon) \quad \text { for } i=L
\end{array}\right.
$$

for $j=1,2, \ldots, E$. If an energy packet is generated for the above case, then we have

$$
p_{(i, j),(1, j)}= \begin{cases}\lambda p\left(1-P_{\text {fail }}\right) \varepsilon & \text { for } i=1,2, \ldots, L-1 \\ \lambda p \varepsilon & \text { for } i=L\end{cases}
$$

for $j=1,2, \ldots, E$.

Finally, the state transition probabilities for trivial state transitions are obtained as

$$
p_{(i, j),(i, j)}=1-\sum_{(k, \ell) \neq(i, j)} p_{(i, j,),(k, \ell)}
$$

for $i=0,1, \ldots, L$ and $j=0,1, \ldots, E$.

\section{Steady-State Probabilities}

Let us denote the steady-state probability of State $(i, j)$ by $\pi_{(i, j)}$ for $i=0,1, \ldots, L$ and $j=0,1, \ldots, E$. Then, according to the theory of Markov chains, the steady-state distribution $\left\{\pi_{(i, j)}\right\}$ can be obtained by solving a system of linear equations;

$$
\left[\begin{array}{c}
\vdots \\
\pi_{(m, n)} \\
\vdots
\end{array}\right]=\left[\begin{array}{ccc} 
& \vdots & \\
\cdots & p_{(i, j),(m, n)} & \cdots \\
& \vdots &
\end{array}\right]\left[\begin{array}{c}
\vdots \\
\pi_{(i, j)} \\
\vdots
\end{array}\right]
$$

and

$$
\sum_{i=0}^{L} \sum_{j=0}^{E} \pi_{(i, j)}=1
$$

\section{Performance Analysis}

\section{A. Fixed Point Equation}

Let $\tau$ denote a ratio of transmitting nodes tentatively. Since a backlogged node with one or more energy packets transmits its data packet with probability $p$, we have

$$
\tau=p \sum_{i=1}^{L} \sum_{j=1}^{E} \pi_{(i, j)}
$$

As shown in the previous subsection, the steady-state distribution $\left\{\pi_{(i, j)}\right\}$ is a function of the probability of transmission failure $P_{\text {fail }}$, which can be formulated as

$$
P_{\text {fail }}=1-(1-\tau)^{N-1}
$$

from the assumption of the independent operation of nodes underlaid in EPA. Here, a combination of (14) and (15) together with (1)-(13) provides a fixed point equation with respect to $\tau$ for given $N, L, E, \lambda, \varepsilon$, and $p$, which can be numerically solved.

Once we obtain the value of $\tau$, we can evaluate various performance measures as follows.

\section{B. Throughput}

The offered traffic is the average number of nodes which are transmitting their data packet in a slot. It follows from the independent operation assumption of nodes that

$$
G=N \tau \text {. }
$$

Then, we can evaluate the throughput as the average number of successful nodes per slot;

$$
S=\left(1-P_{\text {fail }}\right) G=N \tau(1-\tau)^{N-1} .
$$

\section{Average Transmission Delay}

According to Little's result [7], the average transmission delay can be obtained as the ratio of the average number of backlogged nodes to the average number of nodes departing from the backlogged states. In the steady-state, the average number of backlogged nodes is given as

$$
B=N \sum_{i=1}^{L} \sum_{j=0}^{E} \pi_{(i, j)}
$$

Nodes can depart from the backlogged states due to successful data packet transmission or discard of their data packet experiencing an excessive transmission failures. The average number of successful nodes per slot is given by (17). On the other hand, a data packet is discarded, if data transmission from a node in State $(L, j)$ results in failure for $j=1,2, \ldots, E$. The average number of discarded packets per slot is evaluated as

$$
N_{d}=N p P_{\text {fail }} \sum_{j=1}^{E} \pi_{(L, j)} .
$$

Therefore, we can obtain the average transmission delay as

$$
D=\frac{B}{S+N_{d}} \text {. }
$$




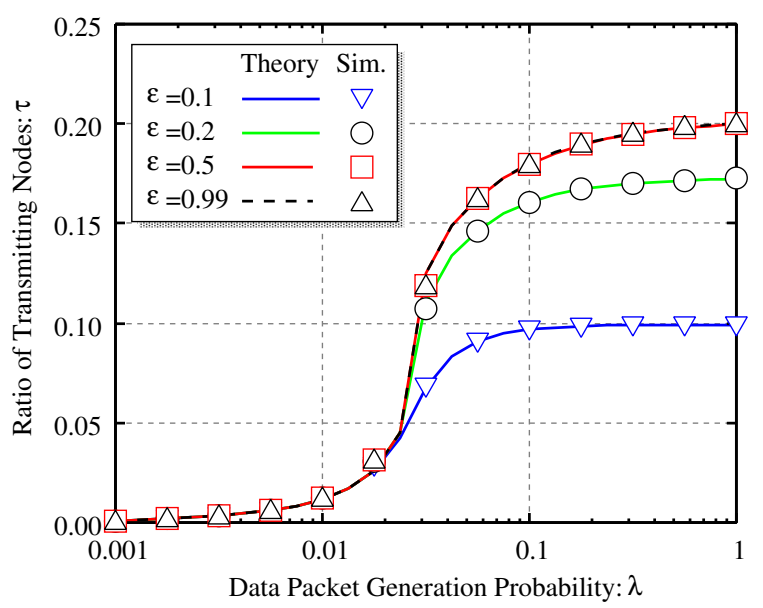

Fig. 3. Comparison of the ratio of transmitting nodes between the fixed point analysis and computer simulation for $N=20, L=20, E=5$ and $p=0.2$.

\section{Discard Probability of Data Packet}

When we impose the retry limit on a data packet, the probability that a generated data packet is discarded is important performance measure. A data packet is released from data buffer at a node because of successful transmission or compulsory discard. Thus, the ratio

$$
P_{d}=\frac{N_{d}}{S+N_{d}}
$$

provides the discard probability of a data packet.

\section{NumericAl RESUlts}

\section{A. Accuracy Verification}

Since EPA assumes the independent operation of nodes, it is required to verify the accuracy of the derived results. As shown in the previous section, various performance measure can be evaluated from the numerical result of $\tau$ obtained by solving the fixed point equation (14). Here, we examine the accuracy of our analysis via the ratio of transmitting nodes $\tau$.

The analytical and computer simulation results are shown in Fig. 3 for $N=20, L=20, E=5$ and $p=0.2$. From Fig. 3 it is clear that the analysis using EPA offers sufficiently accurate numerical results. Also, it can be found that the ratio of transmitting nodes is independent of $\varepsilon \geq 0.5$

\section{B. Performance Measure}

Based on the fixed point equation (14), the numerical results for $N=20, L=20, E=5$ and $p=0.2$ in terms of throughput (17), the offered traffic (16), the average transmission delay (20) and the discard probability of data packet (21) are shown in Fig. 4, Fig. 5 Fig. 6 and Fig. 7, respectively, as a function of the data packet generation probability $\lambda$ and the energy packet generation probability $\varepsilon$.

From Fig. 4, we can observe that the shape of throughput surface exhibits a weak symmetric relationship between data packet generation probability $\lambda$ and energy packet generation probability $\varepsilon$. That is, we can recognize that throughput

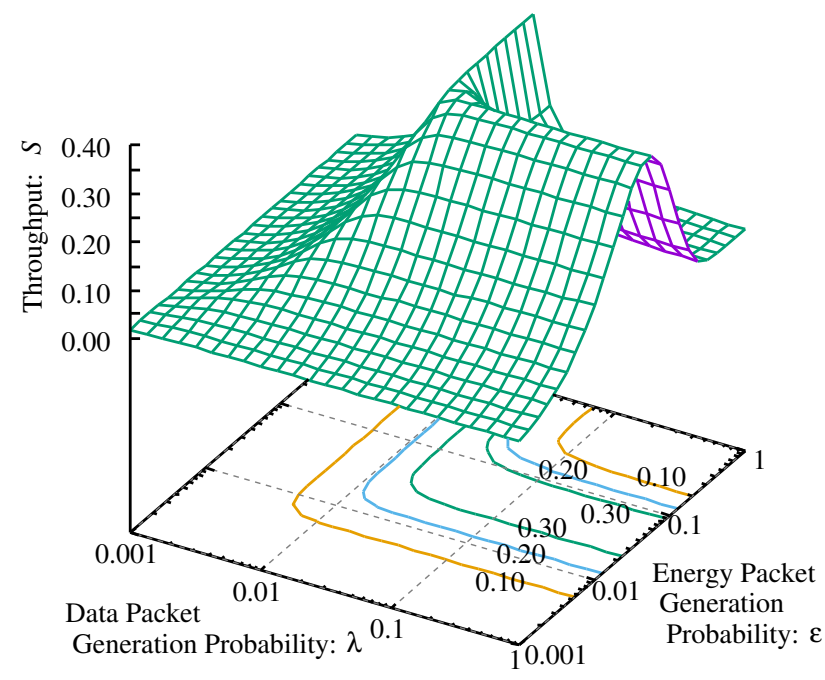

Fig. 4. Throughput for $N=20, L=20, E=5$ and $p=0.2$.

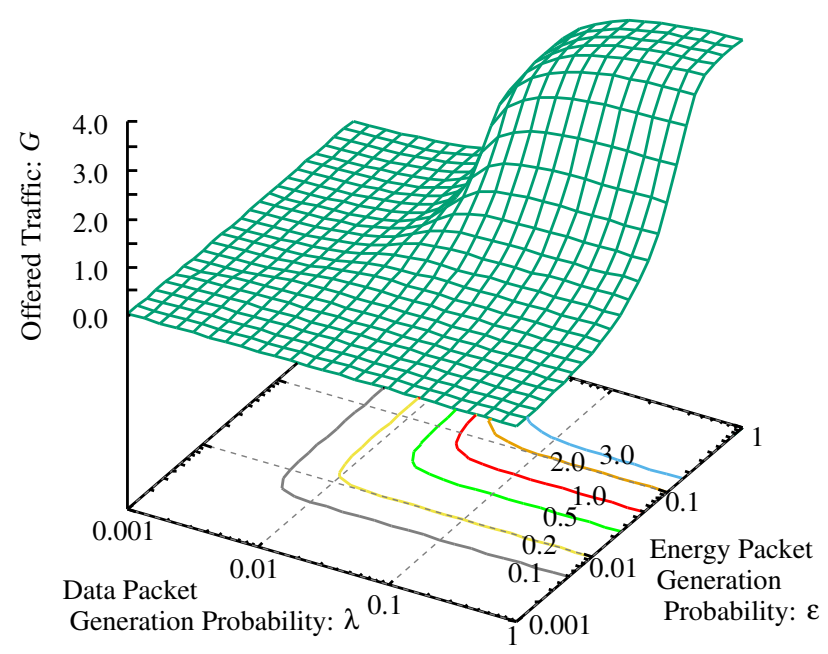

Fig. 5. Offered traffic for $N=20, L=20, E=5$ and $p=0.2$.

roughly depends on $\min [\lambda, \varepsilon]$. A backlogged node can transmit no data packet, unless it has one or more energy packets. Conversely, a node with empty data buffer can transmit no data packet, even if it has one or more energy packets. This relationship results in weak symmetry of throughput between $\lambda$ and $\varepsilon$. It is widely confirmed that throughput of slotted ALOHA systems is maximized when the offered traffic is one data packet per slot. In fact, it follows from Fig. 5 that the offered traffic which achieves the maximum throughput in Fig. 4 is around one data packet per slot; $G \approx 1.0$. Comparing Fig. 5 to Fig. 4, we can find that the shape of the contours projected on the $\lambda-\varepsilon$ plain is closely related. Also, we can perceive that the well-known relationship $S=G e^{-G}$ approximately holds between Fig. 4 and Fig. 5.

Next, from Fig. 6, both the shapes of the surface of the average transmission delay and the contours on the $\lambda-\varepsilon$ plain differ from those of those in Fig. 4 and Fig. 5. We can observe no symmetry between $\lambda$ and $\varepsilon$. Transmission delay 


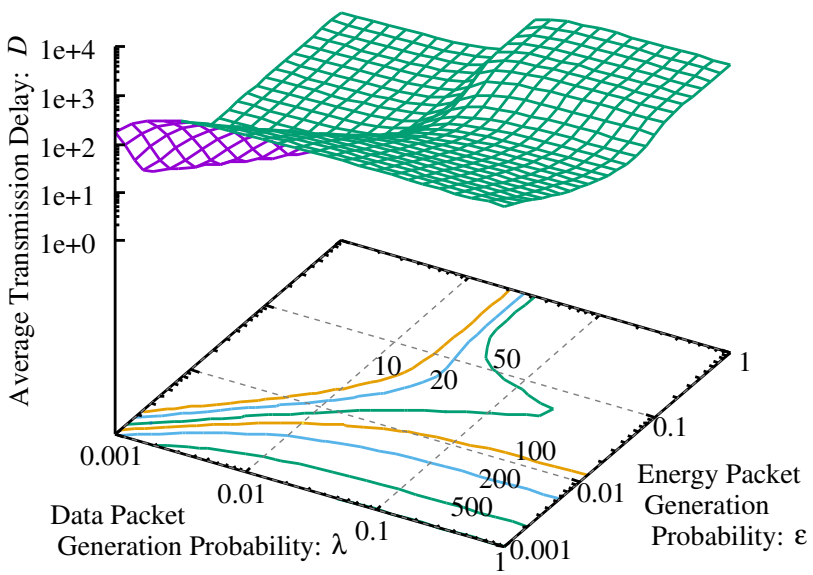

Fig. 6. Average transmission delay of data packet for $N=20, L=20$, $E=5$ and $p=0.2$.

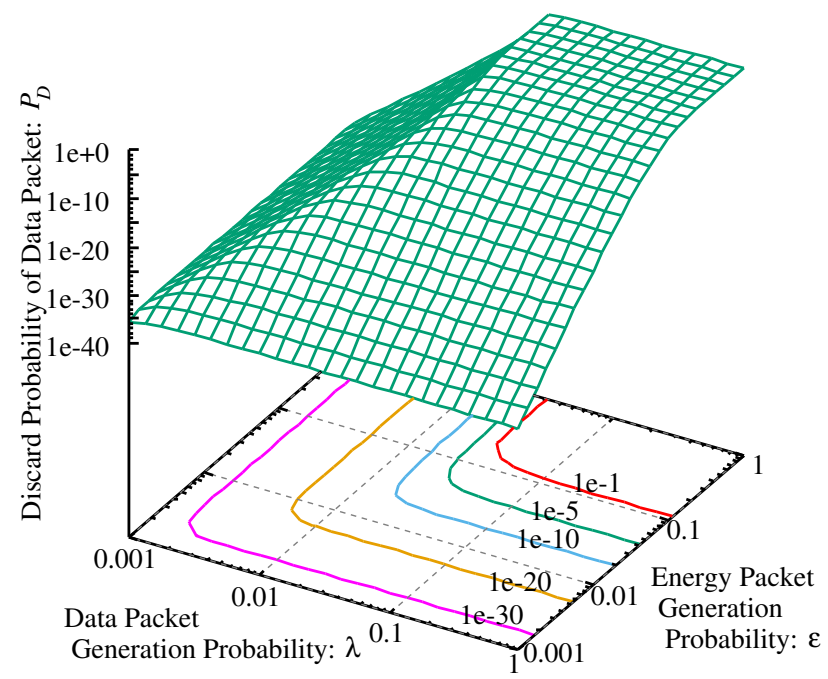

Fig. 7. Discard probabiltiy of data packet for $N=20, L=20, E=5$ and $p=0.2$.

is defined as the elapsed time-slots between the data packet generation and its departure from the system due to successful transmission or discard. Hence, transmission delay increases for small $\varepsilon$, since the generated data packet has little chances to be transmitted due to the lack of the energy despite the data packet generation probability $\lambda$. For small $\lambda$ the average transmission delay tends to decrease according to an increase of $\varepsilon$. This is because the data packet has more chances to be transmitted for large $\varepsilon$ and it has less possibility to collide with other simultaneously transmitted data packets because of small $\lambda$. For given $\lambda>0.05=1 / N$, the average transmission delay has its minimum at around $\varepsilon=0.05=1 / N$. For small $\varepsilon<0.05$, the average transmission delay increases due to less chances for transmission. On the other hand, for $\varepsilon>0.05$, increment of the offered traffic, as shown in Fig. 5, results in more packet collisions, so that the average transmission delay is enlarged.

Finally, the contour of the discard probability of data packet
$P_{D}$ in Fig. 7 exhibits the same tendency as throughput in Fig. 4 and the offered traffic in Fig. 5. However, in contrast to Fig. 4 and Fig. 5, the discard probability is rapidly degraded several orders of magnitude against small fluctuation of $\lambda$ and $\varepsilon$ even if $\lambda$ and $\varepsilon$ are sufficiently small.

\section{CONCLUSION}

In this paper, we analyzed the performance of slotted ALOHA systems consisting of energy harvesting nodes with retry limit. We assumed that the capacities of data and energy buffer at a node are one packet and $E$ packets, respectively, and that one data packet transmission consumes one energy packet. The data and the energy packet arrival processes are modeled as independent and identically distributed Bernoulli processes. Under these assumptions, we developed a nodecentric two-dimensional discrete-time Markov chain model, whose states represent a node state described by a two-tuple of the number of data packets in the data buffer and the number of energy packets in the energy buffer. According to the concept of the equilibrium point analysis, the fixed point equation with respect to the ratio of nodes transmitting a data packet was derived.

Based on the numerical results obtained from the fixed point equation, we derived expressions of throughput, the offered traffic, the average transmission delay and the discard probability of data packet. We verified the theoretical results by means of computer simulation. The numerical results indicated that throughput, the offered traffic and the discard probability roughly depend on the minimum of the data packet generation probability and the energy packet generation probability.

Generalization and relaxation of the assumption such as an independent property of the energy packet arrival process are left for further investigation.

\section{REFERENCES}

[1] H. H. R. Sherazi, L. A. Grieco and G. Boggia, "A comprehensive review on energy harvesting MAC protocols in WSNs: Challenges and tradeoffs," Ad Hoc Networks, vol. 71, pp. 117-134, Mar. 2018. DOI: 10.1016/j.adhoc.2018.01.004.

[2] M. Moradian and F. Ashtiani, "Throughput analysis of a slotted Aloha-based network with energy harvesting nodes," in Proc. IEEE PIMRC 2012, Sydney, Australia, pp. 351-356, Sep. 2012. DOI: $10.1109 /$ PIMRC.2012.6362809.

[3] S. Foss, D. Kim and A. Turlikov, "Model with common energy harvesting for the random multiple access system," in Proc. REDUNDANCY 2014, St. Petersburg, Russia, pp. 39-42, June 2014. DOI: 10.1109/RED.2014.7016701.

[4] Y. H. Bae, "Modeling timely-delivery ration of slotted Aloha with energy harvesting," IEEE Commun. Lett., vol. 21, no. 8, pp. 1823-1826, Aug. 2017. DOI: 10.1109/LCOMM.2017.2693998.

[5] K. Sakakibara, H. Muta and Y. Yuba, "The effect of limiting the number of retransmission trials on the stability of slotted ALOHA systems," IEEE Trans. Veh. Tech., vol. 49, no. 4, pp. 1449-1453, July 2000. DOI: $10.1109 / 25.875281$.

[6] S. Tasaka, "Stability and performance of the R-ALOHA packet broadcast system," IEEE Trans. Comput., vol. C-32, no. 8, pp. 717-726, 1983. DOI: $10.1109 /$ TC.1983.1676309.

[7] M. E. Woodward, Communication and Computer Networks, IEEE Computer Society Press, Los Angeles, CA, 1994.

[8] G. Bianchi, ""Performance analysis of the IEEE 802.11 distributed coordination function," IEEE J. Select. Areas Commun., vol. 18, no. 3, pp. 535-547, 2000. DOI: 10.1109/49.840210. 